\title{
二次谐波在二维材料结构表征中的应用
}

\author{
夏芳芳 ${ }^{1}$, 王发坤 ${ }^{1}$, 胡海龙 ${ }^{2}$, 许 翔 $^{1}$, 李 阳 ${ }^{1}$, 翟天佑 ${ }^{1}$
}

(1. 华中科技大学 材料成形与模具技术国家重点实验室, 材料科学与工程学院, 武汉 430074; 2 . 威泰克(北京)科学 技术有限公司，北京 100027)

摘 要: 二次谐波作为非线性光学的重要分支, 逐渐成为表征晶体结构的重要手段之一。在众多表征方法中, 二次 谐波因其无损检测、高稳定性、可调谐性、超快响应、偏振敏感性、通用性、操作简单等特点被广泛应用于二维 材料结构表征, 为二维材料的物性研究和功能应用提供了重要信息, 大大推动了二维材料基础研究的快速发展。本 文综述了近几年二次谐波在二维材料结构表征中的研究，简述了二次谐波产生原理，介绍了飞秒激光器接入共聚 焦拉曼光谱仪产生二次谐波测试装置, 分别讨论了二次谐波在二维材料的层间堆垛层数、层间堆垛角度、单层二维 材料晶界及晶体取向表征方面的应用。同时, 本文还介绍了采用二次谐波强度直接、灵敏地检测晶体中应变幅度以 及通过二次谐波信号变化跟踪材料中的缺陷变化, 接着讨论了二次谐波与拉曼光谱、光致发光的多维度关联分析在 材料全面深度表征方面的重要性。最后展望了二次谐波未来在材料结构表征中的潜在研究方向。

关 键 词: 二次谐波; 二维材料; 非线性光学; 结构表征; 综述

中图分类号: TQ174 文献标志码: A

\section{Application of Second Harmonic Generation in Characterization of 2D Materials}

\author{
XIA Fangfang ${ }^{1}$, WANG Fakun ${ }^{1}$, HU Hailong ${ }^{2}$, XU Xiang ${ }^{1}$, LI Yang $^{1}$, ZHAI Tianyou ${ }^{1}$
}

(1. State Key Laboratory of Materials Processing and Die \& Mould Technology, School of Materials Science and Engineering, Huazhong University of Science and Technology, Wuhan 430074, China; 2. WITec (Beijing) Scientific Technology Co., Ltd., Beijing 100027, China)

\begin{abstract}
As an important branch of nonlinear optics, second harmonic generation (SHG) is becoming one of the most important means to characterize crystal structure. Among various methods of characterization, because of nondestructive detection, high stability, tunability, ultrafast response, polarization sensitivity, versatility and simplicity, SHG is widely used to characterize the structure of two-dimensional (2D) materials. It provides important information for the physical properties and functional applications of 2D materials, as well as greatly promotes the rapid development of basic research on 2D materials. Here, the current state of the art focuses on the recent research work of SHG in 2D material structure characterization. Firstly, the principle of the second harmonic generation is briefly introduced. Then, the second harmonic generation device with femtosecond laser connected to confocal Raman spectrometer is taken as an example to present the mechanism of SHG. Afterwards, the applications of SHG are demonstrated in the thickness of interlayer stacking of 2D materials, the stacking angle between
\end{abstract}

收稿日期：2021-02-05; 收到修改稿日期：2021-03-26; 网络出版日期：2021-06-01

基金项目: 国家自然科学基金(21825103) National Natural Science Foundation of China (21825103)

作者简介: 夏芳芳(1986-), 女, 工程师. E-mail: xiafangfang@hust.edu.cn XIA Fangfang(1986-), female, engineer. E-mail: xiafangfang@hust.edu.cn

通信作者：翟天佑，教授. E-mail: zhaity@hust.edu.cn ZHAI Tianyou, professor. E-mail: zhaity@hust.edu.cn 
different layers of 2D materials, the grain boundary and the crystal orientation of monolayer 2D materials. The second harmonic intensity is used as a direct and sensitive means to monitor the strain amplitude, and the SHG signal changes are used to track defects in materials. Meanwhile, the importance of multi-dimensional correlation analysis of second harmonic generation, Raman spectroscopy and photoluminescence in comprehensive and in-depth characterization of materials is also explored. Finally, the potential research directions and prospects based on SHG in material characterization in the future are prospected.

Key words: second harmonic generation; two dimensional materials; nonlinear optics; structural characterization; review

二维材料因其区别于体相的超薄原子结构、大 的比表面积和量子限域效应等受到了人们的广泛关 注 $^{[1-4]}$ 。如何高分辨表征二维材料的晶相、晶格取 向、堆垛方式及缺陷等对于其改进合成工艺、提高 材料物性研究和性能应用是至关重要的。目前，二 维材料高分辨精细结构通常利用电子显微技术和扫 描探针显微镜进行表征分析，如扫描电子显微镜 SEM、透射电子显微镜 TEM、扫描隧道电子显微镜 STM 等, 由于其超高的放大倍数以及电子成像原理, 引领研究人员探究材料高分辨精细结构。而光学显 微技术对样品形态、测试环境无严格要求, 且具有 操作简单、测试成本低、无损检测等优点, 成为电 子显微镜进行高分辨微结构表征的有益补充。二次 谐波(Second Harmonic Generation, SHG) 是近年发 展起来的一种光学显微表征技术, 基于光与具有较 大极化率的非中心对称物质的非线性相互作用, 二 次谐波显微成像技术由于其无光损伤、无光毒性、 无光漂白等特点, 近来广泛应用于生物组织结构的 无创检测 ${ }^{[5-7]}$, 如生物组织内原纤维的取向检测 ${ }^{[8]}$ 、 肿瘤基质胶原的检测 ${ }^{[9]}$ 等; 另外, 各种微纳结构中 的二次谐波产生具有信号稳定、波长可调谐、相干 性好、超快响应性和偏振敏感性等独特优点, 作为 新型微纳相干光源, 在显微成像、精密探测和非线性 微纳光学器件等领域有重要的应用前景 ${ }^{[10-15]}$ 。其中, 二次谐波结合共聚焦光学成像技术获得空间光学衍 射极限分辨率在二维材料晶格取向、晶界和堆垛等 结构表征方面发挥了重要作用 ${ }^{[16-19]}$, 为科研工作者 深入分析二维材料微观结构、物理性质等提供了重 要参考 ${ }^{[2,20-21]}$ 。由于二次谐波的产生并不依赖光子吸 收过程, 光子与材料作用时保持能量守恒, 避免了 测试过程对材料结构的破坏; 二次谐波测量不需要 对材料进行特殊处理, 可以简化实验过程并避免外 源性处理对材料结构的影响 ${ }^{[22]}$ 。

本文将在二次谐波产生的原理和基本条件的基 础上, 以飞秒脉冲激光接入共聚焦拉曼显微测试系
统为参考介绍二次谐波产生的实验装置和测试方法, 并着重讨论二次谐波在二维材料堆垛、晶界、异质 结、应力和缺陷表征中的优势与前沿进展，同时也 探索二次谐波与拉曼光谱、光致发光的多维度关联 分析在材料全面深度表征方面的重要性。

\section{1 二次谐波产生的基本原理}

非线性光学效应是指物质与强光相互作用时, 材料的非线性极化导致光响应与光场强度呈非线性 关系, 并产生各种非线性光学现象, 包括和频、差 频、三次谐波、饱和吸收等。二次谐波是和频现象的 一种，即基频入射光与材料相互作用后产生二倍频出 射光 ${ }^{[23]}$, 二次谐波产生原理示意图如图 1 所示。

非线性介质的光学响应可以通过介质的电极化 强度 $P$ 与入射辐射的光场强度 $E$ 来确定, 介质在外 加光电场作用下的极化强度可以表示为:

$$
P=P^{(1)}+P^{(2)}+P^{(3)}+\cdots=\varepsilon_{0}\left(\chi^{(1)} E+\chi^{(2)} E^{2}+\chi^{(3)} E^{3}+\cdots\right)
$$

其中, $\varepsilon_{0}$ 表示真空介电常数, $\chi^{(i)}$ 代表 $i$ 阶非线性光学 系数, 表示非线性材料的极化性质和非对称性, $E$ 代 表光电场的强度。 $\chi^{(1)}$ 为一阶线性极化率, 用于表述 传统的线性光学性质, 如折射、散射和吸收; $\chi^{(2)}$ 代表 二阶极化率，反映了二阶非线性光学效应，如差频、 和频和倍频等。其中目前应用最广泛的是倍频，即 二次谐波，是指在强电场作用下，具有相同频率的 两个光子与非线性介质相互作用后, 产生具有初始 光子两倍能量的新光子, 其频率加倍, 波长减半。二 次谐波过程并不发生能量跃迁, 两个频率为 $\omega$ 的入

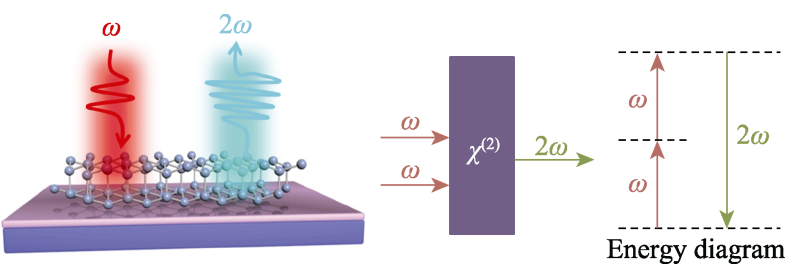

图 1 二次谐波产生原理示意图 ${ }^{[24]}$

Fig. 1 Schematic diagram illustrating the principle of $\mathrm{SHG}^{[24]}$ 
射光子的能量等于一个频率为 $2 \omega$ 发射光子的能量。 根据能量守恒, 介质分子的量子力学状态并不会发 生改变, 即被激发样品不会吸收能量。二次谐波不 仅与光电场强度 $E$ 有关, 还与介质的二阶极化率 $\chi^{(2)}$ 有关。二次谐波的强度可以简化表示为:

$$
\vec{P}=\chi^{(2)}: \vec{E} \vec{E}
$$

其中, $\vec{P}$ 为电介质中二次谐波的电极化强度矢量, $\vec{E}$ 是基频入射光强度矢量 ${ }^{[25]}$ 。

在进行空间反演对称操作时, 二阶电极化强度 矢量和基频入射光强度矢量都改变符号, 而计算出 的电极化强度保持不变, 则 $\chi^{(2)}=0$ 。即对于具有中 心反演对称性的介质, 其二阶极化率为零, 若材料 具有二次谐波现象, 则反映了其中心反演对称性是 被破坏的 ${ }^{[23]}$ 。因此, 可以通过二次谐波现象表征材 料的结构对称性信息。

对于打破中心反演对称性的介质，其二次谐波 的强度 $P_{2 \omega}$ 和频率转化效率 $\eta$ 可以分别通过下式表示:

$$
\begin{gathered}
P_{2 \omega}=\frac{8 \pi^{2} d^{2}}{\varepsilon_{0} c \lambda^{2} A} \cdot \frac{\left[\chi^{(2)}\right]^{2}}{n_{\omega}^{2} n_{2 \omega}} \cdot P_{\omega}^{2} \\
\eta=\frac{P_{2 \omega}}{P_{\omega}}
\end{gathered}
$$

其中, $P_{2 \omega}$ 和 $P_{\omega}$ 分别为倍频和基本激光功率; $\varepsilon_{0}$ 和 $c$ 分别为真空中的介电常数和光速; $A$ 为入射激光光 斑的面积; $\lambda$ 为入射光波长; $d$ 为样品的厚度; $n_{2 \omega}$ 和 $n_{\omega}$ 分别为样品在倍频和基本激光频率下的折射率。 当 $n_{2 \omega}=n_{\omega}$ 时, 满足相位匹配条件, 二次谐波的转 化效率与入射光场和非线性介质的作用长度的平方 成正比 ${ }^{[23]}$ 。当入射光电场取向为 $\hat{e}_{\omega}$ 时, 产生的二次 谐波在 $\hat{e}_{2 \omega}$ 出射方向的强度可以用式(5)描述:
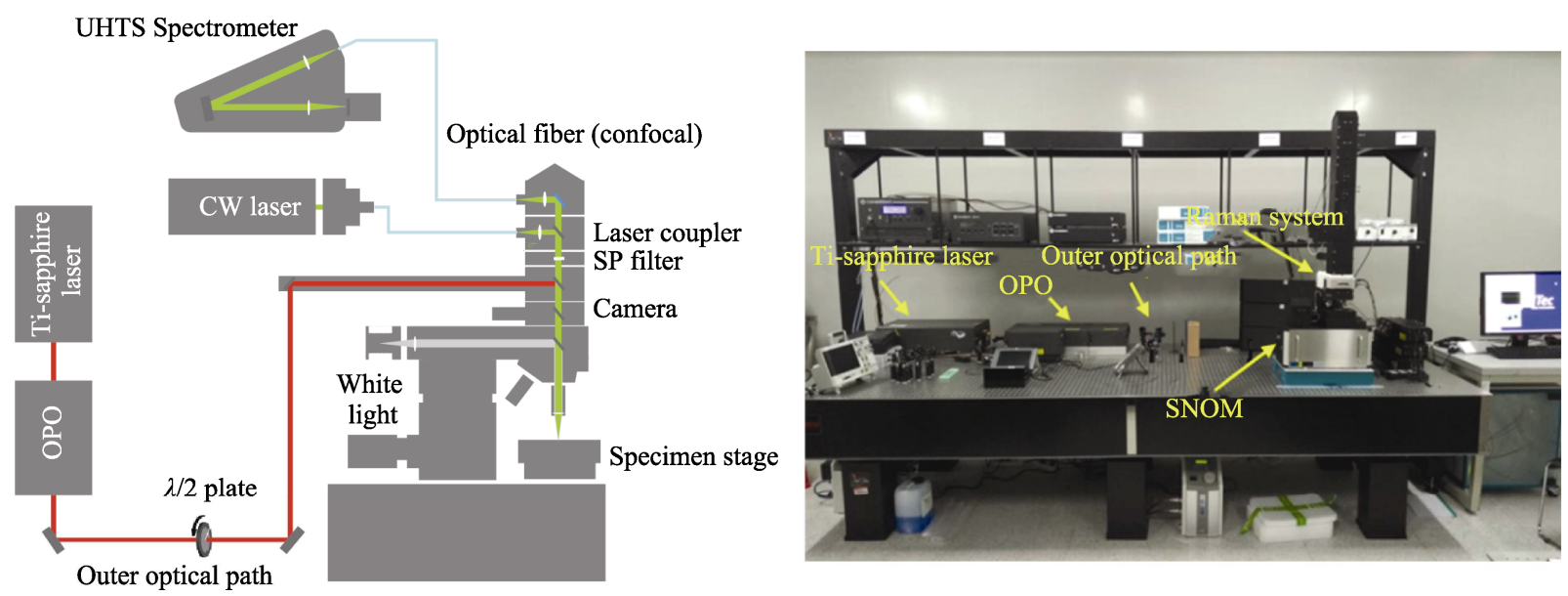

图 2 二次谐波产生装置示意图和实物图

Fig. 2 Schematic diagram and picture of the experimental setup of SHG

$$
I_{\mathrm{SHG}}=\left|\hat{e}_{2 \omega} \cdot d_{\mathrm{eff}} \cdot \hat{e}_{\omega}^{2}\right|^{2}
$$

其中, $d_{\mathrm{eff}}$ 定义为有效二阶非线性系数, $d_{\mathrm{eff}}=$ $1 / 2 \chi^{(2)}$ 。

\section{2 飞秒脉冲激光接入共聚焦拉曼显微 测试系统}

本文以飞秒脉冲激光接入共聚焦拉曼显微测试 系统, 介绍二次谐波的产生装置和测试方法。采用 美国相干公司 Chameleon Ultra II fs 激光器产生的脉 冲激光, 脉冲宽度 $140 \mathrm{fs}$, 重复频率 $80 \mathrm{MHz}$, 输出 波长范围 680 1600 $\mathrm{nm}$ 。将飞秒激光通过自由光路 引入到 alpha 300R 共聚焦拉曼显微镜, 具体地, 激光 通过光参量振荡器调制, 经过一个可调衰减片调节 激光功率和一系列光学组件后, 进入 WITec alpha300RS+共聚焦拉曼显微镜, 最后被显微镜头 (100 倍)聚焦为直径 $1.8 \mu \mathrm{m}$ 的光斑; 二次谐波产生 的信号通过同一显微镜头收集, 并通过一个低通高 滤的滤波片进入拉曼光谱仪。对于角度依赖的二次 谐波, 为确保入射激光为线偏光, 在入射光路中增 设偏振片和半波片。同时, 为检测二次谐波沿特定 方向偏振的强度, 在收集光路中也增设偏振片, 使 得偏振方向与入射偏振方向一致。

该系统扩展了共聚焦拉曼光学显微镜自身的功 能与应用, 将二次谐波非线性成像与光学图像、拉 曼图像以及光致发光成像进行联用, 对于更全面了 解与掌握样品的结构对称性、晶格取向、原子层堆 垛、晶界等性质起到了推动作用。二次谐波产生装 置示意图和实物图如图 2 所示。 


\section{3 二次谐波表征二维层状材料的堆垛}

二维层状材料的结构对称性可以分成两类: 非 中心对称性和中心对称性 ${ }^{[26-31]}$ 。二次谐波对于少层 二维材料的晶体对称性异常敏感 ${ }^{[26-27,29,32-38]}$ 。对于 非中心对称性材料, 其本征具备二次谐波信号, 如

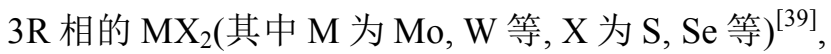
$1 \mathrm{~T}^{\prime \prime \prime}$ 相 $\mathrm{MoS}_{2} 、 \mathrm{InSe} 、 \mathrm{GeTe}$ 等。对于中心对称结构, 如 $2 \mathrm{H}$ 相的 $\mathrm{MX}_{2}$, 随着原子层数的变化, 其所属的空间 群发生变化, 二次谐波信号将产生波动性变化, 对 于奇数层数的 $2 \mathrm{H}$ 相, 由于奇数层的结构转变, 二次 谐波信号的增强, 对于偶数层的 $2 \mathrm{H}$ 相则二次谐波 信号泯灭。因此, 通过二次谐波表征材料的不同堆 垛方式、堆垛层数、层间堆垛角度, 信号会表现出 不同的相对强度。Zeng 等 ${ }^{[40]}$ 通过二次谐波表征不同 相的 $\mathrm{WS}_{2}$, 得到截然不同的层数与二次谐波信号强 度的依赖关系。对于二次谐波信号强度呈现振荡趋 势改变的二维 $\mathrm{WS}_{2}$ (图 3(d)), 其单层为非中心对称 结构, 二次谐波信号较强, 对于两层 $\mathrm{WS}_{2}$, 二次谐 波信号泯灭，这种信号强度的反差可以归因为第二 层 $\mathrm{S}$ 原子和 $\mathrm{W}$ 原子都堆垛于第一层相应原子的正上 方, 两层的 $\mathrm{WS}_{2}$ 恢复了反演对称结构。在中心对称 偶数 $2 \mathrm{~N}$ 层中, 相邻层产生的 $\mathrm{SH}$ 场相互抵消, 导致 没有二次谐波信号。对于奇数层, 只在 $(2 \mathrm{~N} \pm 1)$ 层中 产生残留的二次谐波信号。根据这种二次谐波信号 呈现出振荡减小的趋势能够定性表征少层二维材料 的相对厚度。随着样品层数增加依次增强的二次 谐波信号(图 3(e)), 表明各层均为非中心对称结构, 层间堆垛方向由 $\mathrm{AB}$ 堆垛改变为非中心对称性堆垛, 在平面内发生位移导致面内非线性偶极子和
非零的二阶非线性极化率，不同层的非线性偶极子 相互叠加, 形成逐渐增强的二次谐波信号, 对于这 种少层的二维材料, 通过拟合发现 $I_{\mathrm{SHG}}$ 正比于层数 $N$ 的平方，这和 $3 \mathrm{R}$ 相少层二维材料 $\mathrm{WSe}_{2}$ 的二次谐 波信号强度与层数拟合结果一致 ${ }^{[40]}$ 。Zhao 等 ${ }^{[41]}$ 通 过二次谐波表征了不同相 $\mathrm{MoS}_{2}$ 也得到了层数与二 次谐波信号强度的依赖关系: 对于 $2 \mathrm{H}$ 相，随着层数 增加二次谐波信号产生波动性变化, 奇数层数表现 出二次谐波信号增强，偶数层的 $2 \mathrm{H}$ 相则表现出二 次谐波信号泯灭; 对于 $3 \mathrm{R}$ 相 $\mathrm{MoS}_{2}$, 随着层数增加 二次谐波信号强度依次增强 ${ }^{[41]}$ 。这与 $3 \mathrm{R}$ 和 $2 \mathrm{H}$ 相 $\mathrm{WS}_{2}$ 二次谐波信号强度随层数变化的结论基本一 致。因此，根据二次谐波信号的相对强度，在明确了 结构对称性的基础上，能够进一步对层间堆垛方式 进行表征，根据二次谐波信号强度的变化趋势能够 定性表征少层二维材料的相对厚度。

除了表征二维材料堆垛层数, 由于对晶体取向 的敏感特性，二次谐波能够进一步表征原子层间堆 垛角度。 $\mathrm{Hsu}$ 等 ${ }^{[42]}$ 人工堆垛得到层间扭曲的 $\mathrm{MoS}_{2} / \mathrm{MoS}_{2} 、 \mathrm{WSe}_{2} / \mathrm{MoS}_{2} 、 \mathrm{WSe}_{2} / \mathrm{WS}_{2}$ 双层薄膜, 通 过二次谐波的强度结合偏振来表征层间堆垛角度 (图 4)。当双层堆垛角度改变时，二次谐波强度也发 生明显改变。其中, 当二维材料的扶手椅方向与入 射激光偏振方向 $\vec{E}(\omega)$ 间的夹角分别为 $\varphi_{1} 、 \varphi_{2}$ 时，则 第一层和第二层产生的二次谐波 $\vec{E}_{1}(2 \omega) 、 \vec{E}_{2}(2 \omega)$ 与入射激光偏振 $\vec{E}(\omega)$ 间的角度分别为 $3 \varphi_{1} 、 3 \varphi_{2}$, 双 层堆垛区域的二次谐波电场 $\vec{E}_{\mathrm{s}}(2 \omega)$ 则是 $\vec{E}_{1}(2 \omega)$ 、 $\vec{E}_{2}(2 \omega)$ 的矢量和: $\vec{E}_{\mathrm{s}}(2 \omega)=\vec{E}_{1}(2 \omega)+\vec{E}_{2}(2 \omega)$ 。双层堆 垛区域总的二次谐波强度正比于 $\left.\vec{E}_{\mathrm{s}}(2 \omega)\right|^{2}$, 用二次
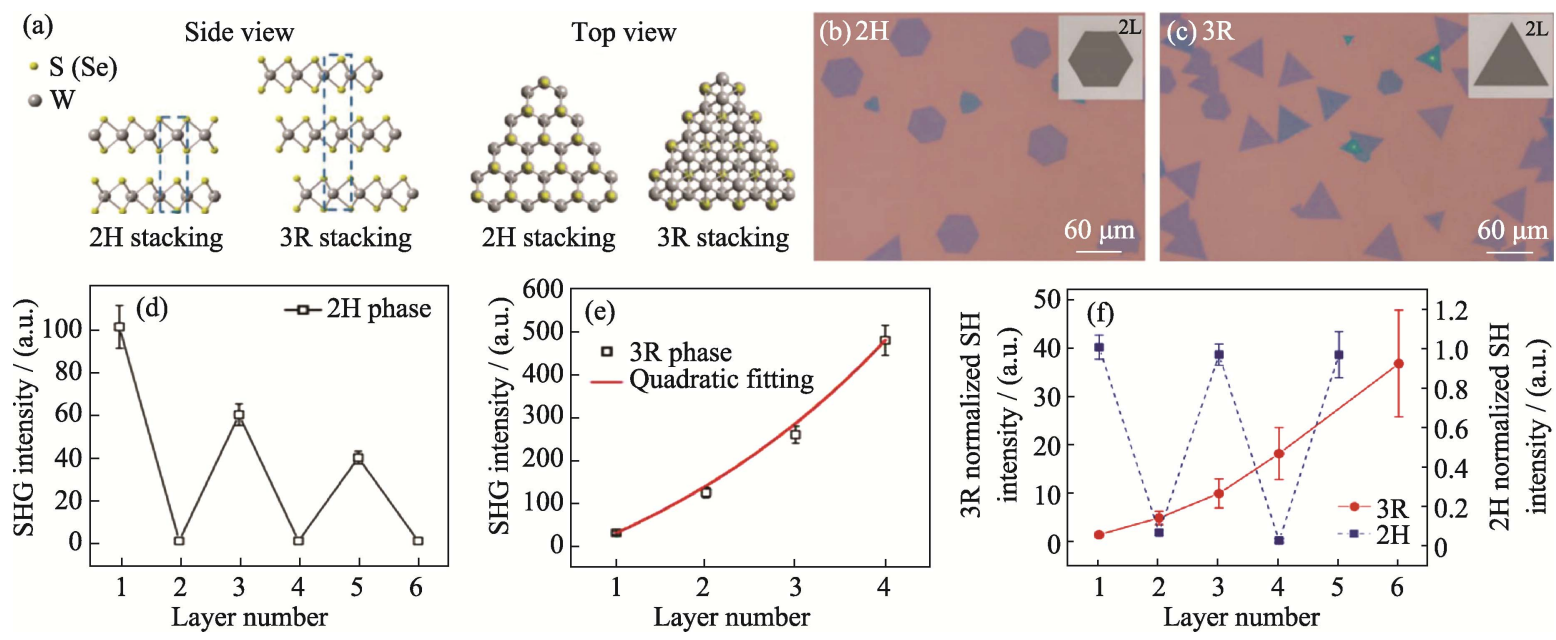

图 3 二次谐波表征不同堆垛层数的二维层状晶体

Fig. 3 Characterization of 2D layered crystals with different stacking layers by SHG

(a) Side view and top view of atomic structure illustration of typical layered $\mathrm{WS}_{2}$ with $2 \mathrm{H}$ and $3 \mathrm{R}$ stacking; $(\mathrm{b}, \mathrm{c})$ Optical images of $2 \mathrm{H}$ and $3 \mathrm{R}$ phase $\mathrm{WS}$; (d,e) SHG intensity of $2 \mathrm{H}$ and $3 \mathrm{R}$ phase $\mathrm{WS}_{2}$ as a function of layer numbers ${ }^{[40]}$; (f) $\mathrm{SHG}$ intensity of $2 \mathrm{H}$ and $3 \mathrm{R}$ phase $\mathrm{MoS}_{2}$ as a function of layer numbers ${ }^{[4]}$ 
(a)

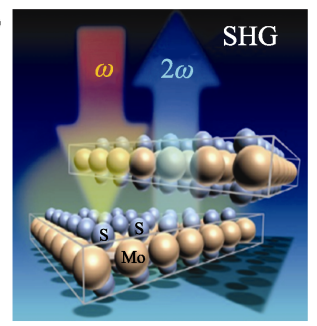

(b)

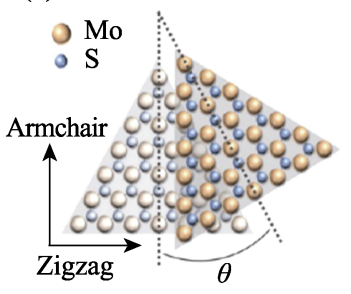

(c)

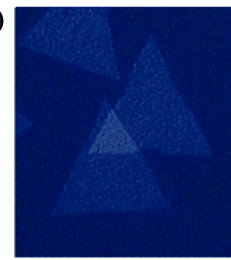

$\theta=2^{\circ}$

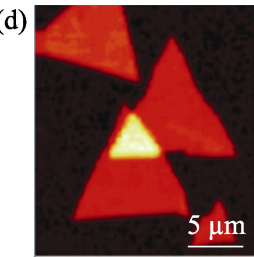

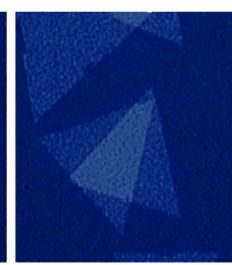

$\theta=30^{\circ}$

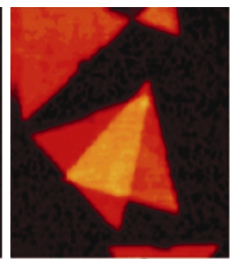

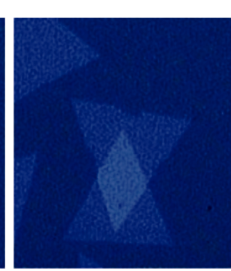

$\theta=54^{\circ}$

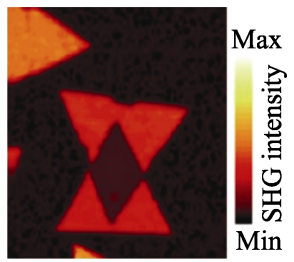

图 4 二次谐波表征层间堆垛角度

Fig. 4 Characterization of stacking angle between layers by SHG (a) Schematic of SHG process in bilayer thin film; (b) Atomic structure of artificially stacked bilayer; (c-d) Optical images for stacked bilayers with different stacking angles and their corresponding SH intensities ${ }^{[42]}$

谐波强度表示为: $I_{\mathrm{s}}(\theta)=I_{1}+I_{2}+2 \sqrt{I_{1} I_{2}} \cos 3 \theta$, 其中 $I_{\mathrm{s}} 、 I_{1} 、 I_{2}$ 分别为堆垛区域、第一单层和第二单 层的二次谐波强度, $\theta$ 为第一层和第二层间的堆垛 角度，等于 $\varphi_{1} 、 \varphi_{2}$ 的差值。当两层的堆垛角度在 $0^{\circ} \sim 60^{\circ}$ 之间变化时, $I_{\mathrm{s}}$ 呈周期性改变, 当 $\theta=0^{\circ}$ 时, 堆垛区域的二次谐波强度是两个单层相长干涉, 相 当于 $3 \mathrm{R}$ 相堆垛, $I_{\mathrm{s}}$ 最大。当 $\theta=60^{\circ}$ 时, 堆垛区域的 二次谐波强度是两个单层相消干涉, 相当于 $2 \mathrm{H}$ 相 堆垛, 导致 $I_{\mathrm{s}}$ 最小 ${ }^{[42]}$ 。因此, 根据这种双层堆垛二 维材料二次谐波极化方向的矢量叠加原理, 通过堆 垛区域的二次谐波强度的相对强弱, 可以进一步表 征二维材料堆垛层之间的角度。

\section{4 二次谐波精准定位二维材料晶界}

由于晶体表面对称性破坏能够形成二维电子态, 对于单层的二维晶体, 晶体边缘的电子结构变化会 产生较强的非线性光学敏感度, 从而通过二次谐波 能够对二维材料的原子边缘和边界直接成像。另外, 通过施加偏振洜浦光, 也可以在大范围内快速、全 光学地确定二维材料的晶体取向。Yin 等 ${ }^{[43]}$ 研究了 CVD 生长的大面积六方二硫化钼 $\left(\mathrm{MoS}_{2}\right)$ 薄膜一维 边缘和边界上的二阶非线性光学。由于反转

对称性被破坏, $\mathrm{MoS}_{2}$ 薄膜呈现出强烈的二次谐波现 象。同时，相邻区域的单层之间存在将不同晶粒连 接在一起的几个原子宽的线缺陷, 即晶界, 导致非 线性波的相消干涉与湮灭。因此，二次谐波成像可 以区分晶粒和晶界。相比于均匀的光学图像(图 5(a)), 二次谐波图像(图 5(b)) 揭示了均匀单层的多晶性质。 每个晶粒内均匀的二次谐波强度表明其是单晶。然 而, 在晶界处, 由于具有不同取向的相邻晶粒产生 的二次谐波相消干涉和湮灭，二次谐波基本上被抑 制。虽然晶界宽度只有几个原子位，但晶界清晰可 见。通过测量边界处的二次谐波强度, 分析二次谐 波的偏振成分，还可以确定两个相邻晶体之间的夹 角, 进而得到多晶单层 $\mathrm{MoS}_{2}$ 的晶体取向图(图 $5(\mathrm{c})$ )。这种方法能够捕捉到完整的晶粒结构图, 根 据晶体方向进行颜色编码 ${ }^{[3]}$ 。针对可视化显示对比 度较弱, 或需要使用化学溶剂来增强图像对比度的 问题, Carvalho 等 ${ }^{[44]}$ 进一步引入暗场二次谐波来增 强显示单层 $\mathrm{MoSe}_{2}$ 的晶界和边缘, 通过暗场二次谐 波能够有效分离发射光成分, 并利用不同取向晶畴 的干涉效应，通过二次谐波发射将晶界和边缘定位 为非常明亮的一维图案, 使晶界和边缘处更加清晰 和直观 ${ }^{[44]}$ 。

二次谐波图像在可视化表征晶界的同时，还能 有效地对晶粒的形成机理进行表征 ${ }^{[45]}$ 。 $\mathrm{Hu}$ 等 ${ }^{[24]}$ 通 过 CVD 方法生长出二维 Ge 晶体, 利用 WITec alpha300RS+显微共聚焦多场联用系统实现对样品 的光学图像和二次谐波成像同区域表征。从光学图 像(图 6(a)) 知两个三角形 $\mathrm{Ge}$ 薄片连为一体, 不存在 明显边界, 无法区分单晶或者两个晶粒; 二次谐波 成像则能够明显分辨出晶界和晶畴(如图 6(b)): 当
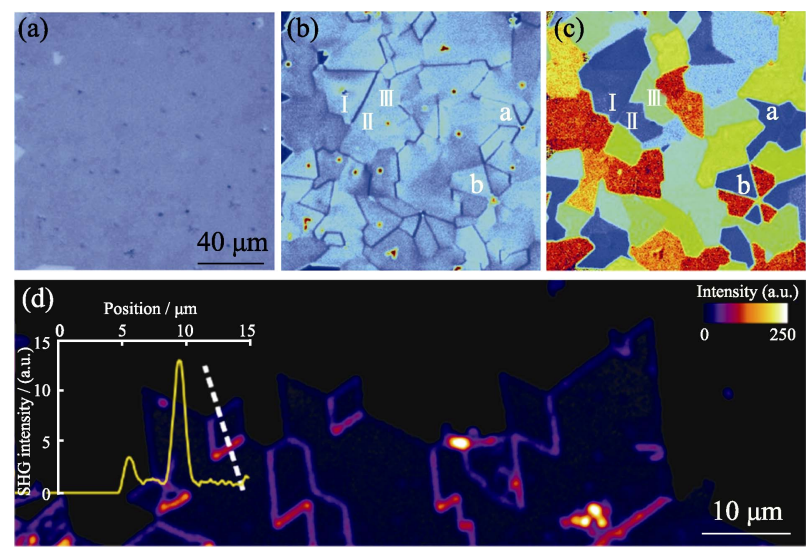

图 5 二次谐波表征二维材料晶界及晶体取向

Fig. 5 Characterization of the grain boundary and crystal orientation of two-dimensional materials by SHG

(a-b) Optical image and SHG image of a polycrystalline monolayer of $\mathrm{MoS}_{2}$ of the same area; (c) Polarized-SHG image showing the crystal orientations ${ }^{[43]}$; (d) Dark-field SHG imaging of a monolayer $\mathrm{MoSe}_{2}{ }^{[44]}$ 

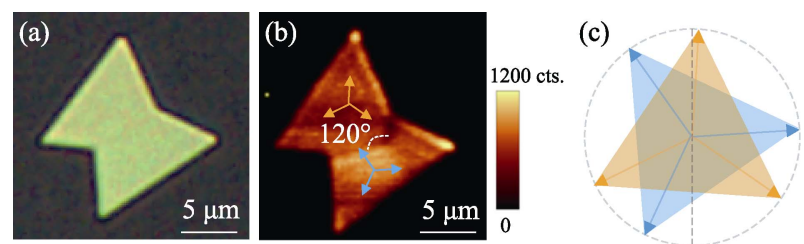

图 6 二次谐波表征晶界及晶粒的形成机理

Fig. 6 Characterization of the grain boundary by SHG and analysis of grain formation mechanism

(a) Optical image and SHG mapping of the flake with boundary; Inset: illustration of the two edges growth at the boundary; (c) Illustration of the armchair directions of the two grains ${ }^{[24]}$

来自相邻晶粒的信号相消干涉时，在二次谐波图像 中清晰可见较暗的分界线, 如图 6(b)所示。而且偏 振二次谐波测试可以得到两个晶畴的晶体取向之间 的夹角(如图 6(c))。在确定了两个 $\mathrm{Ge}$ 晶粒存在晶界 和晶粒取向后, 由于晶体生长过程中相邻边缘的边 界方向取决于相邻边缘的相对生长速率 ${ }^{[45]}$, 根据两个 晶粒接触并继续生长时形成的对称倾斜边界，揭示了 两个晶粒相似的边以相同的间距合并，随后边界将 两条边平分，从而形成对称的倾斜边界的生长机制。

\section{5 二次谐波检测应力和缺陷}

对二维层状材料施加外力产生的应变能够有 效、可逆地调控二维材料的物理和化学性质, 如带 隙、相图和反应活性。但是对局部点应变矢量进行
完整表征仍然面临挑战。对于非中心对称的二维材 料, 由于二次谐波光场强度与非线性光学系数成正 比，二次谐波强度对 $2 \mathrm{D}$ 的结构变化异常敏感。当非 中心对称的二维材料产生应变时，其晶格结构随之 发生改变, 进而引起非线性光学系数的变化。因此, 理论上，采用二次谐波能够原位、灵敏且无损地测 量非中心对称二维材料局部应变。Liang 等 ${ }^{[6]}$ 采用 二次谐波对单层 $\mathrm{MoSe}_{2}$ 单点应变强度、应变方向进 行了表征(图 7(b))。对于单层 $\mathrm{MoSe}_{2}$, 通过改变基底 的形变量获得不同的 $\mathrm{MoSe}_{2}$ 应变, 并采集不同应变 下的二次谐波强度, 得到 $\frac{\Delta I_{\varepsilon}}{I_{0}}=\frac{I_{\varepsilon}-I_{0}}{I_{0}}$, 其中 $I_{\varepsilon}$ 和 $I_{0}$ 分别是应变和无应变下的二次谐波强度, 也即二 次谐波强度随应变呈线性改变, 且斜率比 PL 峰值 强度的相对变化大一个数量级。因此, 通过监测二 次谐波强度变化可以更加灵敏地对应变进行调控。 另外, Mennel 等 ${ }^{[47]}$ 用两点弯曲法对 $\mathrm{MoS}_{2}$ 施加不同 的单轴拉伸应变, 通过光弹性张量建立起应变和非 线性磁化率之间的关系 ${ }^{[48]}$, 测量偏振分辨二次谐波 信号强度，通过三个偏振倍频测量点确定局部应变 张量, 实现高效快速的应变场成像(图 7(d,e)), 补充 和扩展了已有的光学应变测量方法, 使用这种技术, 可以在亚皮秒时间尺度上表征瞬态晶体变形 ${ }^{[47]}$ 。 因此，基于二次谐波对于应变的灵敏反应，二次谐 波强度可以直接、灵敏地监测应变幅度。
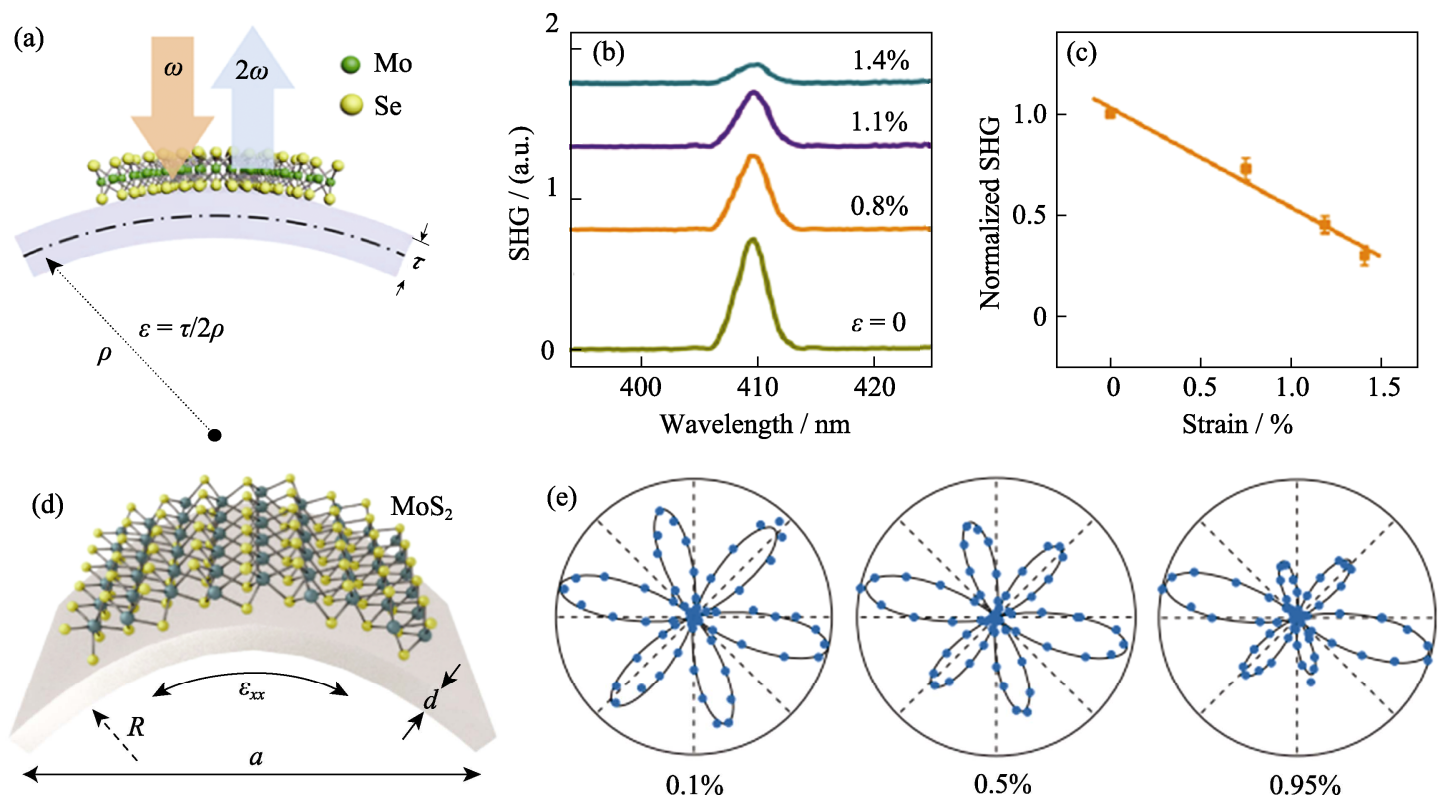

图 7 二次谐波表征二维材料应变

Fig. 7 Characterization of the strain of 2D materials by SHG

(a) Schematic of strain apparatus and SHG process in monolayer $\mathrm{MoSe}_{2}$ under uniaxial tensile strain;

(b) SHG spectra under different strain; (c) Evolution of normalized SHG intensity with strain ${ }^{[46]}$;

(d, e) Schematic illustration of two-point bending method and

SHG patterns for applied tensile strains of $0.1 \%, 0.5 \%$, and $0.95 \%{ }^{[47]}$ 
此外, 随着材料缺陷的引入, 材料的性能也得 以控制或调整 ${ }^{499-50]}$ 。通常采用 STEM、PL 等表征材 料缺陷。近来，二次谐波也被用于单层材料缺陷的 可视化表征。对于缺乏反转对称性的单层过渡金属 二硫化物, 二次谐波信号的变化可用于跟踪材料中 的缺陷密度, 对于衍射受限的激光束, 空间分辨率 通常能够达到亚微米量级。Rosa 等 ${ }^{[51]}$ 通过化学气相 沉积制备了单层 $\mathrm{WS}_{2}$, 并用二次谐波可视化表征其 缺陷，二次谐波信号随材料中缺陷的密度而变化， 对于单层 $\mathrm{WS}_{2}$, 二阶非线性极化率可能会由于缺陷 相关的中能隙态的存在而在红外波段得到改善, 进 而用来区分低缺陷区和高缺陷区(图 8)。在缺陷区, 硫原子被空位取代，二次谐波信号得到增强，高达 2 个数量级。密度函数理论计算也表明, 这种与空位 相关的缺陷在材料的能带结构中产生了中能隙态, 当 $\mathrm{WS}_{2}$ 的光学带隙为 $1.9 \mathrm{eV}$ 时, 中能隙态距离价带 顶部约 $1.5 \mathrm{eV}$ ，使得基波 $(1560 \mathrm{~nm}$ )的倍频(780 nm) 成为准共振过程，因此，在 $780 \mathrm{~nm}$ 处的二次谐波图 像能够清晰直观地区分出高低缺陷区，在缺陷密度 高的区域，二次谐波得到显著增强，进而实现高缺

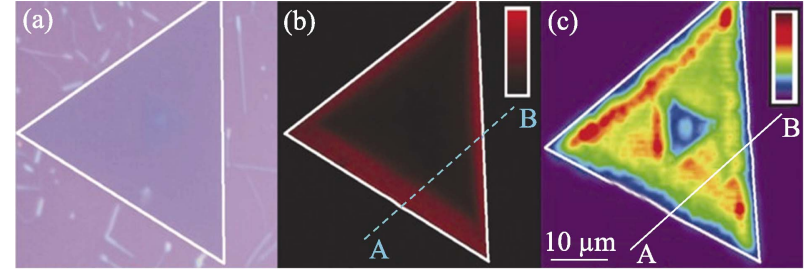

图 8 二次谐波表征二维材料的缺陷

Fig. 8 Characterization of the defects of 2D materials by SHG (a-c) Optical image, fluorescence image and second harmonic mapping image of monolayer $\mathrm{WS}_{2}{ }^{[51]}$

陷密度区域的可视化识别 ${ }^{[51]}$ 。因此，通过二次谐波 信号的变化来跟踪材料中的缺陷，能够快速、无损 地表征材料的缺陷区域。

\section{6 二次谐波与拉曼光谱、光致发光光}

\section{谱的多维度关联分析}

集成了非线性光学表征功能的共聚焦拉曼显微 测试系统可以实现同区域的拉曼、光致发光(Photoluminescence, PL)和二次谐波非线性光学成像的联
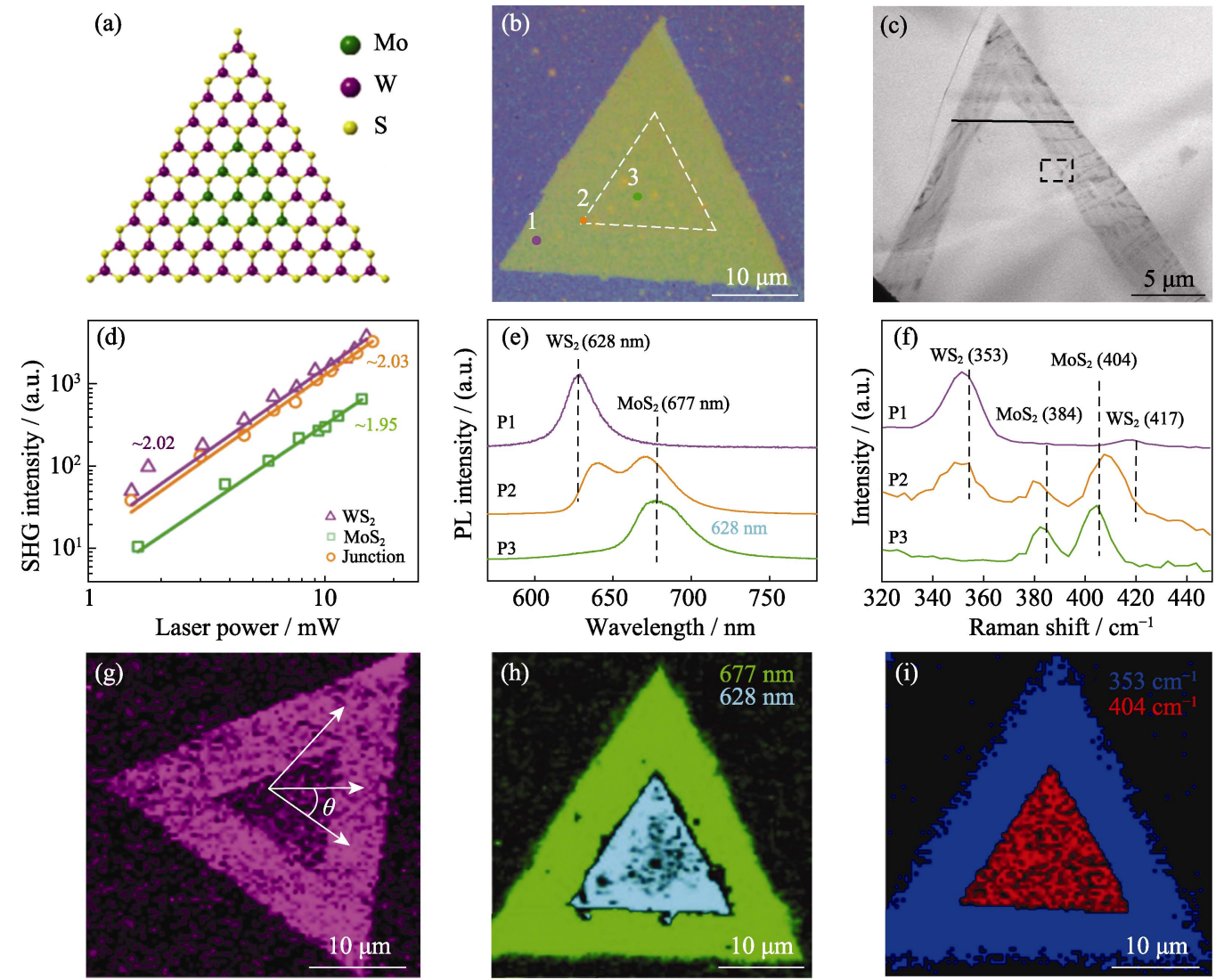

图 9 二次谐波与光致发光、拉曼光谱联合表征二维材料 $\left(\mathrm{MoS}_{2} / \mathrm{WS}_{2}\right.$ 异质结 $)$

Fig. 9 Characterization of $2 \mathrm{D}$ materials $\left(\mathrm{MoS}_{2} / \mathrm{WS}_{2}\right.$ heterojunction) by $\mathrm{SHG}$ combined with photoluminescence and Raman spectroscopy

(a) Atomic structure diagram; (b-c) Optical image and TEM image; (d, g) SHG intensity and mapping; $(e, h)$ Photoluminescence spectra at different positions; (f, i) Corresponding Raman spectra ${ }^{[52]}$ 
用, 这能够帮助研究者更全面地了解二维材料样品 的晶体结构。Wu 等 ${ }^{[52]}$ 利用 WITec alpha300RS+显微 共聚焦拉曼成像系统对 $\mathrm{MoS}_{2} / \mathrm{WS}_{2}$ 异质结分别进行 二次谐波、拉曼和 PL 表征。首先, 通过光学图像 (图 9(b))发现合成的二维材料样品为均匀的三角形 结构，进一步采用 TEM 观察异质结构。同时，采集 二次谐波在 $400 \mathrm{~nm}$ 处图像, 均存在较强的二次谐波 响应，且在核壳处存在清晰边界; 但是中间三角形 区域的响应强度低于边缘区域, 进一步证实了异质 结的存在。通过进一步对光学图像中不同位置处进 行 PL 和拉曼表征, PL 光谱(图 9(e))呈现两个特征发 射峰, 外层约 $628 \mathrm{~nm}$ 和内层约 $677 \mathrm{~nm}$ 处出现的峰 值分别归因于 $\mathrm{WS}_{2}$ 和 $\mathrm{MoS}_{2}$ 的近带边发射; 同时, 对应的 PL 映射(图 9(h)) 显示, 核 $\left(\mathrm{MoS}_{2}\right)$ 和壳 $\left(\mathrm{WS}_{2}\right)$ 区域都显示出均匀和明亮的对比度变化, 进一步表 明其元素分布均匀，同时，在异质结界面处，发光 位置呈现出两个特征峰, 且相较于 $\mathrm{MoS}_{2}$ 和 $\mathrm{WS}_{2}$, 峰 位均出现了一定的偏移, 这可归因于 $\mathrm{MoS}_{2}$ 和 $\mathrm{WS}_{2}$ 晶格常数不同导致晶格失配所引起的应变。同区域 拉曼表征进一步证实由该区域产生的两个特征峰属 于单层 $\mathrm{MoS}_{2}$, 在 $404 \mathrm{~cm}^{-1}$ 处为 $\mathrm{A}_{1 \mathrm{~g}}$ 振动模式, 在 $384 \mathrm{~cm}^{-1}$ 处为 $\mathrm{E}_{2 \mathrm{~g}}$ 振动模式; 同时, 在壳区域 417 和 $353 \mathrm{~cm}^{-1}$ 处呈现的两个拉曼峰分别对应于 $\mathrm{WS}_{2}$ 的 $A_{1 g}$ 和 $E_{2 g}$ 振动模式。从异质结界面处收集的光谱同 时包含 $\mathrm{MoS}_{2}$ 和 $\mathrm{WS}_{2}$ 共振模式, 进一步证实了在该 区域两个不同相共存的性质。因此, 通过将二次谐 波表征引入共聚焦拉曼显微成像系统, 采用二次谐 波、PL 和拉曼进行同区域表征，得到的材料结构信 息能够互相补充和相互印证，为更加全面地探知二 维材料的结构信息提供便捷的测试手段。

\section{7 总结和展望}

总之, 二次谐波在二维材料的研究中得到了广 泛的应用，具有对晶体对称性敏感、非接触、无损 伤、无需制备和易于操作等优点。本文着眼于二次 谐波在二维材料结构表征中的应用，介绍了二次谐 波产生机理、测试装置和方法，重点阐述了二次谐 波在二维材料层间堆垛、相结构、晶界、晶体取向、 晶体缺陷等结构表征方面的应用，同时，二次谐波与 拉曼光谱、光致发光的多维度关联分析在材料全面 深度表征方面发挥着重要作用。最后, 二次谐波作 为一种重要的二维材料结构表征手段, 未来将会在 二维材料研究中发挥越来越重要的作用。例如, 对于 新兴的二维磁性材料 ${ }^{[33-55]}$, 采用二次谐波可以进一
步研究磁有序结构、磁畴和超快磁动力学等 ${ }^{[5]}$ 。随着 二维材料的相变引起了研究者广泛关注 ${ }^{[56-57]}$, 未来也 可以采用二次谐波对相变材料的结构转变进行表征。

\section{参考文献:}

[1] WANG Q H, KALANTAR-ZADEH K, KIS A, et al. Electronics and optoelectronics of two-dimensional transition metal dichalcogenides. Nature Nanotechnology, 2012, 7(11): 699-712.

[2] WANG Y, XIAO J, YANG S, et al. Second harmonic generation spectroscopy on two-dimensional materials [Invited]. Optical Materials Express, 2019, 9(3): 1136-1149.

[3] ZHAI T Y, GAN L, WANG R Y. $\operatorname{ReX}_{2}(\mathrm{X}=\mathrm{S}, \mathrm{Se})$ : a new opportunity for development of two-dimensional anisotropic materials. Journal of Inorganic Materials, 2019, 34(1): 1-16.

[4] MENG J H, GAO M L, ZHANG X W. Research progress of direct growth of two-dimensional hexagonal boron nitride on dielectric substrates. Journal of Inorganic Materials, 2019, 34(12): 1245-1256.

[5] CHEN W, OUYANG J, YI X, et al. Black phosphorus nanosheets as a neuroprotective nanomedicine for neurodegenerative disorder therapy. Advanced Materials, 2018, 30(3): 1703458.

[6] HRISTU R, EFTIMIE L G, STANCIU S G, et al. Quantitative second harmonic generation microscopy for the structural characterization of capsular collagen in thyroid neoplasms. Biomed Opt. Express, 2018, 9(8): 3923-3936.

[7] DENG X, WILliAMS E D, THOMPSON E W, et al. Second-harmonic generation from biological tissues: effect of excitation wavelength. Scanning, 2002, 24(4): 175-178.

[8] ODIN C, LE GRAND Y, RENAULT A, et al. Orientation fields of nonlinear biological fibrils by second harmonic generation microscopy. Journal of Microscopy, 2008, 229(1): 32-38.

[9] LIN L, CHEN G, CHEN Z, et al. Prognostic value of tumor stromal collagen features in patients with hepatocellular carcinoma revealed by second-harmonic generation microscopy. Experimental and Molecular Pathology, 2020, 116:104513

[10] SURIYA M, MANI MARAN M, BOAZ B M, et al. Crystal growth, structural, optical and piezoelectric investigations on novel B4AAT (bis-4-acetylanilinium tartrate): a phase matchable second and third-order NLO single crystal for optoelectronic and nonlinear optical device applications. Optical Materials, 2020, 108: 110042.

[11] SIVAKUMAR T, ANBARASAN R, KALYANA SUNDAR J, et al. Enhancing the SHG effect of zinc chloride-doped DAST single crystals: new potential materials for nonlinear optical device applications. Journal of Materials Science: Materials in Electronics, 2020, 31(15): 12943-12954.

[12] BHOWMIK G, AN Y Q, SCHUJMAN S, et al. Optical second harmonic generation from silicon (100) crystals with process tailored surface and embedded silver nanostructures for silicon nonlinear nanophotonics. Journal of Applied Physics, 2020, 128(16): 165106

[13] PACKIYA RAJ M, RAVI KUMAR S M, SIVAVISHNU D, et al. Synthesis, growth and optical, mechanical, electrical and surface properties of an inorganic new nonlinear optical crystal: sodium cadmium tetra chloride (SCTC). Crystal Research and Technology, 2018, 53(2): 1700271.

[14] HONG X, HU G, ZHAO W, et al. Structuring nonlinear wavefront emitted from monolayer transition-metal dichalcogenides. Research, 2020, 2020: 9085782.

[15] PANTAZIS P, MALONEY J, WU D, et al. Second harmonic generating (SHG) nanoprobes for in vivo imaging. Proceeding of National Academy of Sciences of the United States of America, 2010, 107(33): 14535-14540.

[16] YUAN F, SUN S, HUANG Y, et al. Improvement of 
second-harmonic generation induced by structural distortions in $\mathrm{Nb}$ doped $\mathrm{YCa}_{9}\left(\mathrm{VO}_{4}\right)_{7}$ crystals. Journal of Alloys and Compounds, 2017, 726: 860-865.

[17] ZHANG Z, ZHANG L, GOGNA R, et al. Large enhancement of second-harmonic generation in $\mathrm{MoS}_{2}$ by one dimensional photonic crystals. Solid State Communications, 2020, 322: 114043.

[18] YU Y, WANG J, WEI Y M, et al. Precise characterization of self-catalyzed III-V nanowire heterostructures via optical second harmonic generation. Nanotechnology, 2017, 28(39): 395701.

[19] HUANG W J, HOU H Y, CHEN X B, et al. Synthesis of InSe nanoflakes with near-infrared photoresponse grown by chemical vapor deposition. Chemical Research in Chinese Universities, 2020, 41(4): 682-689.

[20] ZENG Z X S, WANG X, PAN A L. Second harmonic generation of two-dimensional layered materials: characterization, signal modulation and enhancement. Acta Physica Sinica, 2020, 69(18): 184210.

[21] YAN C, GAN L, ZHOU X, et al. Space-confined chemical vapor deposition synthesis of ultrathin $\mathrm{HfS}_{2}$ flakes for optoelectronic application. Advanced Functional Materials, 2017(27): 1702918.

[22] WEN X, GONG Z, LI D. Nonlinear optics of two-dimensional transition metal dichalcogenides. InfoMat, 2019, 1(3): 317-337.

[23] 李家泽, 朱宝亮, 魏光辉. 晶体光学. 北京: 北京理工大学出版 社, 1989: 343-390.

[24] HU X, HUANG P, JIN B, et al. Halide-induced self-limited growth of ultrathin nonlayered Ge flakes for high-performance phototransistors. Journal of the American Chemical Society, 2018, 140(40): 12909-12914.

[25] 张克从, 王希敏. 非线性光学晶体材料科学. 北京: 科学出版 社, 2005: 26-77.

[26] SU J, WANG M, LI Y, et al. Sub-millimeter-scale monolayer p-type H-phase $\mathrm{VS}_{2}$. Advanced Functional Materials, 2020, 30(17): 2000240.

[27] HUANG W, GAN L, LI H, et al. Phase-engineered growth of ultrathin InSe flakes by chemical vapor deposition for highefficiency second harmonic generation. Chemistry, 2018, 24(58): $15678-15684$.

[28] YANG D, HU X, ZHUANG M, et al. Inversion symmetry broken 2D 3R-MoTe $e_{2}$. Advanced Functional Materials, 2018, 28(26): 1800785.

[29] WANG F, ZHANG Z, ZHANG Y, et al. Honeycomb $\mathrm{RhI}_{3}$ flakes with high environmental stability for optoelectronics. Advanced Materials, 2020, 32(25): 2001979.

[30] WANG R, LIANG F, WANG F, et al. $\mathrm{Sr}_{6} \mathrm{Cd}_{2} \mathrm{Sb}_{6} \mathrm{O}_{7} \mathrm{~S}_{10}$ : strong SHG response activated by highly polarizable $\mathrm{Sb} / \mathrm{O} / \mathrm{S}$ groups. Angewandte Chemie International Edition, 2019, 58(24): 8078-8081.

[31] LI L, HAN W, PI L, et al. Emerging in-plane anisotropic two-dimensional materials. InfoMat, 2019, 1(1): 54-73.

[32] YU Y, RAN M, ZHOU S, et al. Phase-engineered synthesis of ultrathin hexagonal and monoclinic GaTe flakes and phase transition study. Advanced Functional Materials, 2019, 29(23): 1901012.

[33] DAI M, CHEN H, WANG F, et al. Robust piezo-phototronic effect in multilayer gamma-InSe for high-performance self-powered flexible photodetectors. ACS Nano, 2019, 13(6): 7291-7299.

[34] FANG Y, HU X, ZHAO W, et al. Structural determination and nonlinear optical properties of new $1 \mathrm{~T}^{\prime \prime \prime}$-type $\mathrm{MoS}_{2}$ compound. Journal of the American Chemical Society, 2019, 141(2): 790-793.

[35] GONG C, CHU J, YIN C, et al. Self-confined growth of ultrathin 2D nonlayered wide-bandgap semiconductor $\mathrm{CuBr}$ flakes. Advanced Materials, 2019, 31(36): e1903580.

[36] FENG X, SUN Z, PEI K, et al. 2D inorganic bimolecular crystals with strong in-plane anisotropy for second-order nonlinear optics. Advanced Materials, 2020, 32(32): e2003146.

[37] LI Y, RAO Y, MAK K F, et al. Probing symmetry properties of few-layer $\mathrm{MoS}_{2}$ and h-BN by optical second-harmonic generation. Nano Letters, 2013, 13(7): 3329-3333.

[38] CHEN S Y, GOLDSTEIN T, VENKATARAMAN D, et al. Activation of new Raman modes by inversion symmetry breaking in type II weyl semimetal candidate T'-MoTe 2 . Nano Letters, 2016, 16(9): 5852-5860.

[39] SONG Y, TIAN R, YANG J, et al. Second harmonic generation in atomically thin $\mathrm{MoTe}_{2}$. Advanced Optical Materials, 2018, 6(17): 1701334.

[40] ZENG Z, SUN X, ZHANG D, et al. Controlled vapor growth and nonlinear optical applications of large-area $3 \mathrm{R}$ phase $\mathrm{WS}_{2}$ and $\mathrm{WSe}_{2}$ atomic layers. Advanced Functional Materials, 2019, 29(11): 1806874.

[41] ZHAO M, YE Z, SUZUKI R, et al. Atomically phase-matched second-harmonic generation in a $2 \mathrm{D}$ crystal. Light: Science \& Applications, 2016, 5(8): e16131.

[42] HSU W T, ZHAO Z A, LI L J, et al. Second harmonic generation from artificially stacked transition metal dichalcogenide twisted bilayers. ACS Nano, 2014, 8(3): 2951-2958.

[43] YIN X, YE Z, CHENE D A, et al. Edge nonlinear optics on a $\mathrm{MoS}_{2}$ atomic monolayer. Science, 2014, 344(6183): 488-490.

[44] CARVALHO B R, WANG Y, FUJISAWA K, et al. Nonlinear dark-field imaging of one-dimensional defects in monolayer dichalcogenides. Nano Letters, 2020, 20(1): 284-291.

[45] CHENG J, JIANG T, JI Q, et al. Kinetic nature of grain boundary formation in as-grown $\mathrm{MoS}_{2}$ monolayers. Advanced Materials, 2015, 27(27): 4069-4074.

[46] LIANG J, ZHANG J, LI Z, et al. Monitoring local strain vector in atomic-layered $\mathrm{MoSe}_{2}$ by second-harmonic generation. Nano Letters, 2017, 17(12): 7539-7543.

[47] MENNEL L, FURCHI M M, WACHTER S, et al. Optical imaging of strain in two-dimensional crystals. Nature Communications, 2018, 9(1): 516.

[48] LYUBCHANSKII I L, DADOENKOVA N N, LYUBCHANSKII $\mathrm{M} \mathrm{I}$, et al. Second-harmonic generation from realistic film-substrate interfaces: the effects of strain. Applied Physics Letters, 2000, 76(14): 1848-1850.

[49] CUNHA R, CADORE A, RAMOS S, et al. Second harmonic generation in defective hexagonal boron nitride. Journal of Physics: Condensed Matter, 2020, 32(19): 19LT01.

[50] MURRAY W, LUCKING M, KAHN E, et al. Second harmonic generation in two-dimensional transition metal dichalcogenides with growth and post-synthesis defects. 2D Materials, 2020, 7(4): 045020 .

[51] ROSA H G, JUNPENG L, GOMES L C, et al. Second-harmonic spectroscopy for defects engineering monitoring in transition metal dichalcogenides. Advanced Optical Materials, 2018, 6(5): 1701327.

[52] WU W, ZHANG Q, ZHOU X, et al. Self-powered photovoltaic photodetector established on lateral monolayer $\mathrm{MoS}_{2}-\mathrm{WS}_{2}$ heterostructures. Nano Energy, 2018, 51: 45-53.

[53] JIMENEZ V O, KALAPPATTIL V, EGGERS T, et al. A magnetic sensor using a 2D van der Waals ferromagnetic material. Scientific Reports, 2020, 10(1): 4789.

[54] NAFDAY D, SEN D, KAUSHAL N, et al. 2D ferromagnetism in layered inorganic-organic hybrid perovskites. Physical Review Research, 2019, 1(3): 032034.

[55] DENEV S A, LUMMEN T T A, BARNES E, et al. Probing ferroelectrics using optical second harmonic generation. Journal of the American Ceramic Society, 2011, 94(9): 2699-2727.

[56] XU X, CHEN S, LIU S, et al. Millimeter-scale single-crystalline semiconducting $\mathrm{MoTe}_{2}$ via solid-to-solid phase transformation. Journal of the American Chemical Society, 2019, 141(5): 2128-2134.

[57] LI J, ZHOU P, ZOU Z, et al. Topological phase transition in 2D 1T'-WSTe. Physica Status Solidi (b), 2020, 257(9): 2000010. 